\title{
Equilibrium Properties of A Monomer-Monomer Catalytic Reaction on A One-Dimensional Chain
}

\author{
G. Oshanin ${ }^{1}$, M. N. Popescu ${ }^{2,3}$, and S. Dietrich ${ }^{2,3}$ \\ ${ }^{1}$ Laboratoire de Physique Théorique des Liquides, \\ Université Paris 6, 4 Place Jussieu, 75252 Paris, France \\ ${ }^{2}$ Max-Planck-Institut für Metallforschung, \\ Heisenbergstr. 3, D-70569 Stuttgart, Germany \\ ${ }^{3}$ Institut für Theoretische und Angewandte Physik, \\ Universität Stuttgart, Pfaffenwaldring 57, D-70569 Stuttgart, Germany
}

\begin{abstract}
We study the equilibrium properties of a lattice-gas model of an $A+B \rightarrow 0$ catalytic reaction on a one-dimensional chain in contact with a reservoir for the particles. The particles of species $A$ and $B$ are in thermal contact with their vapor phases acting as reservoirs, i.e., they may adsorb onto empty lattice sites and may desorb from the lattice. If adsorbed $A$ and $B$ particles appear at neighboring lattice sites they instantaneously react and both desorb. For this model of a catalytic reaction in the adsorption-controlled limit, we derive analytically the expression of the pressure and present exact results for the mean densities of particles and for the compressibilities of the adsorbate as function of the chemical potentials of the two species.
\end{abstract}

PACS: 05.50.+q; 64.60.Cn; 68.43.De; 82.65.+r 


\section{Introduction}

During the two decades following the work of Ziff, Gulari, and Barshad (ZGB) [1] there has been a remarkable development in the theoretical analysis of catalytically activated reactions. The ZGB model, sometimes referred to as the "monomer-dimer" model, has been introduced to describe the important process of oxidation of carbon monoxide on a catalytic surface [2]. Within this model, a monomer (CO) adsorbs onto a single vacant site of the surface (since dissociation of $\mathrm{CO}$ is not considered, $\mathrm{CO}$ is treated as a monomer), while a dimer $\left(\mathrm{O}_{2}\right)$ adsorbs onto a pair of adjacent vacant sites and then immediately dissociates. Both $\mathrm{CO}$ and $\mathrm{O}_{2}$ are in thermal contact with their gaseous reservoirs. Nearest neighbors of adsorbates composed of a dissociated $\mathrm{O}$ atom and a $\mathrm{CO}$ molecule react and form a $\mathrm{CO}_{2}$ molecule, which then desorbs from the metal surface. The ZGB model predicts remarkable new features compared to the classical mean-field kinetic schemes of rate equations [2]: for a two-dimensional catalytic substrate as the $\mathrm{CO}$ gas pressure is lowered the system undergoes a first-order transition from a $\mathrm{CO}$ saturated inactive phase (zero rate of $\mathrm{CO}_{2}$ production) into a reactive steady state (non-zero rate of $\mathrm{CO}_{2}$ production) followed by a continuous transition into an $\mathrm{O}_{2}$-saturated inactive phase. This continuous transition was shown to belong to the same universality class as the directed percolation and the Reggeon field theory [3]. A simpler "monomer-monomer" model, in which particles of both species can adsorb on single but different sites, has also been proposed [4-7], and it has been shown that in $(2+1)$ spatial dimensions this system exhibits a first-order transition from a phase saturated with one species to one saturated with the other. Allowing desorption of one species leads to a continuous transition which also belongs to the directed percolation universality class $[7,8]$.

These observations have significantly increased the interest in the properties of such models of catalytically activated reactions. Different aspects of the dynamics of the adsorbed phase for these two models, as well as for their extensions to molecules with more complicated structures (e.g., dimer-dimer [9], dimer-trimer [10] models), have been examined thoroughly [11-23], and the studies have confirmed an essentially collective, many-particle behavior. On the other hand, the equilibrium properties of catalytically activated reactions in systems in which the reactive species undergo continuous exchanges with their vapor phases acting as reservoirs, i.e., adsorb on and desorb from the catalytic substrate, have been much less studied, and, consequently, the understanding of the equilibrium state remains rather limited. Only recently such equilibrium properties, revealing a rather non-trivial behavior, have been obtained for the more simple case of single-species reactions $A+A \rightarrow 0$ on a one-dimensional

chain with a random distribution of catalytic segments or catalytic sites [24,25]. Kinetics of the diffusion-limited $A+A \rightarrow 0$ reactions, controlled by the constraint that the particles may 
undergo reactions only when they meet each other in the vicinity of special catalytic sites has been discussed in Ref. [26].

Here we study the properties of the equilibrium state for a very simple model of a monomer-monomer $A+B \rightarrow 0$ catalytic reaction on a one-dimensional chain. Within this model the particles of species $A$ and $B$ undergo continuous exchanges with the mixed vapor phase, i.e., they adsorb onto empty lattice sites on the chain and may thermally desorb from them, while the vapor phase as a reservoir is steadily maintained at constant chemical potentials. In addition, if any adsorbed $A$ and $B$ particles appear at neighboring lattice sites, they instantaneously react and both leave the chain (i.e., desorb), and the reaction product $A B$ is completely removed from the system. For this model we derive the expression for the pressure of the adsorbed particles, which provides the complete thermodynamical description of the system, and we present exact asymptotic (in respect to the chemical potential) results for the mean densities of the particles and for the compressibilities of the system. We note that one could also consider a model in which the product $A B$ is not removed from the system but is forming its own vapor phase from which it may readsorb on the substrate. However, this more complicated case will not be studied here.

In Sec. 2 we describe the model and introduce basic notations. In Sect. 3 we derive the recursion relations obeyed by the partition function of the system and obtain the solution of these recursion relations. The connection of this model with classical spin $S=1$ models is discussed in Sect. 4. In Sect. 5 we derive the expressions of the mean densities of the particles and of the compressibilities of the system, analyze their asymptotic behavior as function of the chemical potentials, and present results of Monte Carlo simulations for the occupation of the lattice in the limit of infinite chemical potentials. We conclude the paper with a brief summary in Sect. 6.

\section{Model}

Consider a one-dimensional, regular lattice of $N$ adsorption sites (Fig. 1), which is brought in contact with the mixed vapor phase of two types $A$ and $B$ of hard-core particles without attractive interactions. The difference in chemical potential between the vapor phase and the adsorbed phase $\mu_{A}$ and $\mu_{B}$, including the binding energy of an occupied site, is maintained constant (thus $\mu_{A, B}>0$ corresponds to a preference for adsorption), and the constant activities are hence defined by

$$
z_{A}=\exp \left(\beta \mu_{A}\right), \quad z_{B}=\exp \left(\beta \mu_{B}\right),
$$

where $\beta^{-1}=k_{B} T$ is the thermal energy, $T$ is the temperature, and $k_{B}$ is the Boltzmann constant. 


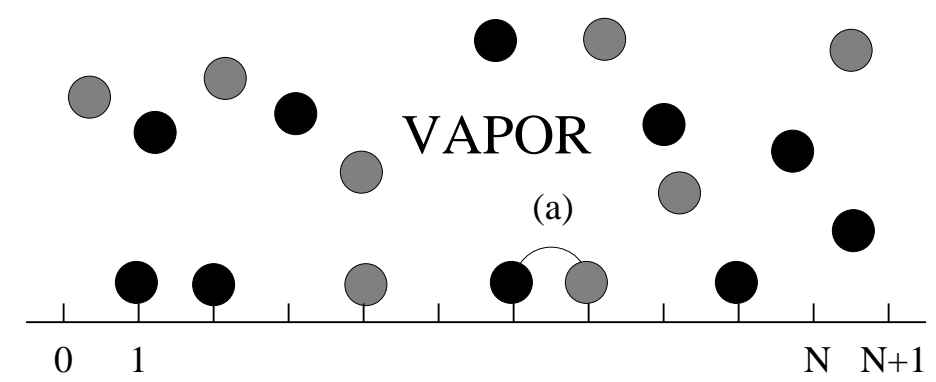

Figure 1: One-dimensional regular lattice of $N$ adsorption sites in contact with a mixed vapor phase. Black and gray circles denote $A$ and $B$ particles, respectively. (a) denotes a configuration in which an instantaneous reaction takes place upon which both particles desorb and the product $A B$ leaves the system. The sites $i=0$ and $i=N+1$ are always empty.

The $A$ and $B$ particles can adsorb from the vapor phases onto vacant sites, and can desorb back to the reservoir. The occupation of the $i$-th site is described by two Boolean variables $n_{i}$ and $m_{i}$, such that

$$
n_{i}=\left\{\begin{array}{l}
1, \text { if the } i \text {-th site is occupied by an } A \text { particle } \\
0, \text { otherwise }
\end{array}\right.
$$

and

$$
m_{i}=\left\{\begin{array}{l}
1, \text { if the } i \text {-th site is occupied by a } B \text { particle } \\
0, \text { otherwise }
\end{array}\right.
$$

and thus the state of the site $i$ is specified by $\left(n_{i}, m_{i}\right) \in\{(0,0),(0,1),(1,0),(1,1)\}$. Note that the state $(1,1)$, which in general is allowed, has a zero probability in the case of hardcore interaction. (The equivalent description in terms of $\sigma_{i}=0,+1,-1$ for empty sites and sites occupied by $A$ and $B$, respectively, will be discussed in Sect. 4.) For computational convenience we add two extra "boundary" sites $i=0$ and $i=N+1$ at both ends of the chain and stipulate that these sites are always unoccupied, i.e., $n_{0}=n_{N+1}=m_{0}=m_{N+1}=0$. Furthermore, the system is locally "frustrated", i.e., the equilibrium between the chain and the reservoir is locally perturbed by the "kinetic" constraint that as soon as an $A$ and a $B$ particle occupy neighboring sites they instantaneously react and leave the chain (and the $A B$ product leaves the system); therefore, our model corresponds to a purely adsorption controlled dynamics [15].

In equilibrium the partition function of this two-species adsorbate on a chain of $N$ sites, in which the configurations $\left\{\left(n_{i}, m_{i}\right)\right\}$ with neighboring $A$ and $B$ or double occupancy of a 
site are excluded, can be written as:

$$
Z_{N}=\sum_{\left\{\left(n_{i}, m_{i}\right)\right\}} \prod_{i=1}^{N} z_{A}^{n_{i}} z_{B}^{m_{i}} \Phi_{i},
$$

where $\Phi_{i}$ is an indicator function with the following properties:

$$
\Phi_{i}=\left\{\begin{array}{l}
1, \text { if site } i \text { is empty, } \\
1, \text { if site } i \text { is occupied by an } A \text { particle, i.e., }\left(n_{i}, m_{i}\right)=(1,0), \text { while } m_{i \pm 1}=0 \\
1, \text { if site } i \text { is occupied by a } B \text { particle, i.e., }\left(n_{i}, m_{i}\right)=(0,1), \text { while } n_{i \pm 1}=0, \\
0, \text { otherwise. }
\end{array}\right.
$$

A very simple realization of the indicator function $\Phi_{i}$ is given by

$$
\Phi_{i}=\left(1-n_{i} m_{i}\right)\left(1-n_{i} m_{i-1}\right)\left(1-n_{i} m_{i+1}\right)\left(1-m_{i} n_{i-1}\right)\left(1-m_{i} n_{i+1}\right) .
$$

The factor $\left(1-n_{i} m_{i}\right)$ ensures that each site $i$ can be occupied by either $A$ or $B$ particle, or be empty, while the product $\left(1-n_{i} m_{i-1}\right)\left(1-n_{i} m_{i+1}\right)$ ensures that if the site $i$ is occupied by an $A$ the neighboring sites are not occupied by a $B$ (and vice versa for the last two terms). Note that $\Phi_{i}$ in Eq. (3) is symmetric with respect to the variables $n_{i}$ and $m_{i}$, and therefore the partition function in Eq. (2) is a symmetric function of $z_{A}$ and $z_{B}$.

The thermodynamics is given by the pressure (in units of the square of the lattice spacing),

$$
P \equiv P\left(T, \mu_{A}, \mu_{B}\right)=\frac{1}{\beta} \lim _{N \rightarrow \infty} \frac{\ln Z_{N}}{N},
$$

from which all other quantities of interest, such as the mean densities of the species $A$ and $B$ and the compressibilities can be obtained straightforwardly by differentiating it suitably with respect to the chemical potentials $\mu_{A}$ and $\mu_{B}$.

Before closing this section we mention two trivial cases:

(a) If the $A$ and $B$ particles do not react and do not interact, such that a lattice site can be occupied by an $A$, a $B$, or simultaneously by an $A$ and a $B$ particle, i.e., $\Phi_{i} \equiv 1$, the pressure and the mean density of the $A(B)$ particles is given by the classical Langmuir adsorption model

$$
P=\frac{1}{\beta} \ln \left(1+z_{A, B}\right) \quad \text { and } \quad n_{A, B}=\frac{z_{A, B}}{1+z_{A, B}} .
$$

(b) If the $A$ and $B$ particles do not react, but obey the hard-core exclusion interaction such that a lattice site cannot accommodate more than one particle (either $A$ or $B$ ), i.e., $\Phi_{i} \equiv\left(1-n_{i} m_{i}\right)$, one has

$$
P=\frac{1}{\beta} \ln \left(1+z_{A}+z_{B}\right) \quad \text { and } \quad n_{A, B}=\frac{z_{A, B}}{1+z_{A}+z_{B}} .
$$

For the following these results will serve as reference expressions, allowing us to identify that contribution to the thermodynamics of the system which stems from the "reaction" part alone. 


\section{Calculation of the partition function based on recursion re- lations}

We define two auxiliary partition functions:

$$
Z_{N}^{(A)}=\left.\sum_{\left\{\left(n_{i}, m_{i}\right)\right\}} \prod_{i=1}^{N} z_{A}^{n_{i}} z_{B}^{m_{i}} \Phi_{i}\right|_{n_{N}=1} \quad \text { and } Z_{N}^{(B)}=\left.\sum_{\left\{\left(n_{i}, m_{i}\right)\right\}} \prod_{i=1}^{N} z_{A}^{n_{i}} z_{B}^{m_{i}} \Phi_{i}\right|_{m_{N}=1}
$$

which are the partition functions of the chain under the constraint that the site $i=N$ is occupied by an $A$ particle (i.e., $n_{N}=1$ ) or a $B$ particle (i.e., $m_{N}=1$ ), respectively. Summing over the occupation variables of the site $i=N$ yields the following relation for the unconstrained partition function (Eq. (2)):

$$
Z_{N}=Z_{N-1}+Z_{N}^{(A)}+Z_{N}^{(B)}, \text { for } N \geq 2
$$

Summing over the occupation variables of the site $i=N-1$ for $n_{N}=1$ or $m_{N}=1$, respectively, one finds that the constrained partition functions (Eq. (7)) satisfy

$$
Z_{N}^{(C)}=z_{C} Z_{N-2}+z_{C} Z_{N-1}^{(C)}, \text { for } N \geq 3, C=A, B
$$

Equations (8) and (9), augmented with the obvious initial conditions

$$
\begin{aligned}
& Z_{1}=1+z_{A}+z_{B}, \\
& Z_{1}^{(A)}=z_{A}, \quad Z_{2}^{(A)}=z_{A}+z_{A}^{2}, \\
& Z_{1}^{(B)}=z_{B}, \quad Z_{2}^{(B)}=z_{B}+z_{B}^{2},
\end{aligned}
$$

represent a closed system of coupled recursion relations determining $Z_{N}$ for arbitrary $N, z_{A}$, and $z_{B}$.

The solution of Eqs. (8)-(10) can be found using the standard generating function technique. Let

$$
\mathcal{Z}_{t}=\sum_{N=1}^{\infty} Z_{N} t^{N}, \quad \mathcal{Z}_{t}^{(C)}=\sum_{N=1}^{\infty} Z_{N}^{(C)} t^{N}, C=A, B
$$

denote such generating functions. Multiplying Eqs. (8) and (9) by $t^{N}$, summing over $N$, and using the expressions in Eq. (10) for the corresponding coefficients of the terms in $t$ and $t^{2}$, we find that $\mathcal{Z}_{t}, \mathcal{Z}_{t}^{(A)}$, and $\mathcal{Z}_{t}^{(B)}$ obey the following equations:

$$
\begin{aligned}
& (1-t) \mathcal{Z}_{t}=t+\mathcal{Z}_{t}^{(A)}+\mathcal{Z}_{t}^{(B)} \\
& \left(1-z_{A} t\right) \mathcal{Z}_{t}^{(A)}=z_{A} t^{2} \mathcal{Z}_{t}+z_{A} t(1+t) \\
& \left(1-z_{B} t\right) \mathcal{Z}_{t}^{(B)}=z_{B} t^{2} \mathcal{Z}_{t}+z_{B} t(1+t) .
\end{aligned}
$$


Consequently, $\mathcal{Z}_{t}$ is a rational function of $t$ and is given explicitly by

$$
\mathcal{Z}_{t}=\frac{t \mathcal{L}_{1}(t)}{\mathcal{L}_{2}(t)}
$$

where

$$
\mathcal{L}_{1}(t)=\frac{1+z_{A}+z_{B}}{z_{A} z_{B}}-2 t-t^{2}, \quad \mathcal{L}_{2}(t)=\frac{1}{z_{A} z_{B}}-\frac{1+z_{A}+z_{B}}{z_{A} z_{B}} t+t^{2}+t^{3} .
$$

Let $t_{1}, t_{2}$ and $t_{3}$ be the roots of $\mathcal{L}_{2}(t)$ so that $\mathcal{L}_{2}(t)=\left(t-t_{1}\right)\left(t-t_{2}\right)\left(t-t_{3}\right)$. Expressing Eq. (13) in terms of elementary fractions and expanding the emerging factors $1 /\left(t_{j}-t\right), j=1,2,3$, into Taylor series in powers of $t / t_{j}$, Eq. (13) can be cast into the form

$$
\mathcal{Z}_{t}=\sum_{N=1}^{\infty}\left\{\alpha^{(1)}\left(\frac{t}{t_{1}}\right)^{N}+\alpha^{(2)}\left(\frac{t}{t_{2}}\right)^{N}+\alpha^{(3)}\left(\frac{t}{t_{3}}\right)^{N}\right\}
$$

where the coefficients $\alpha^{(j)}$ are given explicitly by

$$
\alpha^{(1)}=\frac{t_{1}+t_{2} t_{3}}{\left(t_{1}-t_{2}\right)\left(t_{1}-t_{3}\right)}, \quad \alpha^{(2)}=\frac{t_{2}+t_{1} t_{3}}{\left(t_{2}-t_{1}\right)\left(t_{2}-t_{3}\right)}, \quad \alpha^{(3)}=\frac{t_{3}+t_{1} t_{2}}{\left(t_{3}-t_{1}\right)\left(t_{3}-t_{2}\right)} .
$$

Equations (11) and (15) imply that the partition function of a chain with $N$ sites (Eq. (2)) is given explicitly by

$$
Z_{N}=\frac{\alpha^{(1)}}{t_{1}^{N}}+\frac{\alpha^{(2)}}{t_{2}^{N}}+\frac{\alpha^{(3)}}{t_{3}^{N}}
$$

and thus its behavior is completely determined by the properties of the roots of the cubic polynomial $\mathcal{L}_{2}(t)$. Defining

$$
q=\frac{3+3 z_{A}+3 z_{B}+z_{A} z_{B}}{54 z_{A} z_{B}}, \quad r=\frac{36+9 z_{A}+9 z_{B}+2 z_{A} z_{B}}{9 z_{A} z_{B}}, \text { and } X=\frac{r}{q^{3 / 2}},
$$

one can show that $Q=r^{2}-q^{3}<0$ so that $0<X<1$ for all $z_{A, B}>0$, which implies that all three roots of $\mathcal{L}_{2}(t)$ are real and given by [27]

$$
\begin{gathered}
t_{1,3}= \pm 2 \sqrt{q} \cos \left( \pm \frac{\pi}{6}+\frac{1}{3} \arcsin (X)\right)-\frac{1}{3}, \\
t_{2}=2 \sqrt{q} \sin \left(\frac{1}{3} \arcsin (X)\right)-\frac{1}{3} .
\end{gathered}
$$

The roots of $\mathcal{L}_{2}(t)$ satisfy $t_{1} t_{2} t_{3}=-\frac{1}{z_{A} z_{B}}<0, t_{1} t_{2}+t_{1} t_{3}+t_{2} t_{3}=-\frac{\left(1+z_{A}+z_{B}\right)}{z_{A} z_{B}}<0$, and since all three are real, it follows that one root is negative and the other two are positive. Using $0<\arcsin (X)<\pi / 2$, it is easy to prove that for any $z_{A}$ and $z_{B}$ one has $t_{1}>t_{2}>$ $t_{3}$ (thus $t_{3}$ is the negative solution) and $\left|t_{3}\right|>t_{1}$. Consequently, due to Eq. (4), in the thermodynamic limit $N \rightarrow \infty$ the pressure is given by

$$
P=-\frac{1}{\beta} \ln \left(2 \sqrt{q} \sin \left(\frac{1}{3} \arcsin (X)\right)-\frac{1}{3}\right) .
$$


We note that here three different roots appear since the model under study includes effectively three-site interactions, as shown by the indicator function $\Phi_{i}$ in Eq. (3). The fact that as function of the chemical potentials neither pair of roots does intersect for any $z_{A}$ and $z_{B}$ implies, as expected, that the one-dimensional model considered here does not exhibit a phase transition. More complicated models involving particles which require more than one empty lattice site for their adsorption, such as, for instance, the original ZGB model in which $A$ species are dimers [1], can be treated within essentially the same approach as presented here. Such models would include effective four-site interactions (or more), and thus four or more different roots will emerge.

\section{Connection with classical spin $\mathrm{S}=1$ models}

Since, as we have noted, in the present model the hard-core interaction excludes the state $\left(n_{i}, m_{i}\right)=(1,1)$ for a site, there is a natural connection between the present model and the three-state lattice gas model [28] or, equivalently, a special Blume-Emery-Griffiths (BEG) spin $S=1$ model [29].

The mapping to the BEG model is accomplished as follows. We assign to each site of the one-dimensional lattice a three-state variable $\sigma_{i}, i=1, \ldots, N$, such that

$$
\sigma_{i}=\left\{\begin{array}{cl}
+1, & \text { if the } i \text {-th site is occupied by an } A \text { particle } \\
-1, & \text { if the } i \text {-th site is occupied by a } B \text { particle } \\
0, & \text { if the } i \text {-th site is empty. }
\end{array}\right.
$$

In terms of $\sigma_{i}$ the occupation numbers $n_{i}$ and $m_{i}$ may be rewritten as

$$
n_{i}=\left(\sigma_{i}+\sigma_{i}^{2}\right) / 2, \quad m_{i}=\left(-\sigma_{i}+\sigma_{i}^{2}\right) / 2
$$

Periodic boundary conditions imply $\sigma_{N+1} \equiv \sigma_{1}$. Defining the nearest-neighbor (NN) coupling as (the limits corresponding to our particular model are indicated in parenthesis)

$$
J_{i, j}=\left\{\begin{array}{cl}
-E_{1}(\rightarrow 0), & \text { for A-A neighbors } \\
-E_{2}(\rightarrow 0), & \text { for B-B neighbors } \\
+E_{3}(\rightarrow \infty), & \text { for A-B or B-A neighbors } \\
0, & \text { otherwise }
\end{array}\right.
$$

one can write the Hamiltonian of this system as

$$
\mathcal{H}=\sum_{<i j>}\left[n_{i} n_{j}\left(-E_{1}\right)+m_{i} m_{j}\left(-E_{2}\right)+\left(n_{i} m_{j}+n_{j} m_{i}\right) E_{3}\right]-\sum_{i=1}^{N}\left(\mu_{A} n_{i}+\mu_{B} m_{i}\right)
$$


where $\sum_{<i j>}$ means summation over all pairs of NN sites with each pair included only once. Replacing $n_{i}, m_{i}$ with the corresponding expressions (Eq. (23)) and collecting the terms, the Hamiltonian above may be rewritten as

$$
\begin{aligned}
\mathcal{H}= & -\frac{E_{1}+E_{2}+2 E_{3}}{4} \sum_{<i j>} \sigma_{i} \sigma_{j}-\frac{E_{1}+E_{2}-2 E_{3}}{4} \sum_{<i j>} \sigma_{i}^{2} \sigma_{j}^{2}-\frac{E_{1}-E_{2}}{4} \sum_{<i j>}\left(\sigma_{i} \sigma_{j}^{2}+\sigma_{j} \sigma_{i}^{2}\right) \\
& -\frac{\mu_{A}-\mu_{B}}{2} \sum_{i=1}^{N} \sigma_{i}-\frac{\mu_{A}+\mu_{B}}{2} \sum_{i=1}^{N} \sigma_{i}^{2},
\end{aligned}
$$

i.e., takes the form of a Hamiltonian for a spin $S=1$ model [30] with the parameters

$J=\frac{E_{1}+E_{2}+2 E_{3}}{4}, K=\frac{E_{1}+E_{2}-2 E_{3}}{4}, C=\frac{E_{1}-E_{2}}{4}, H=\frac{\mu_{A}-\mu_{B}}{2}$, and $\Delta=-\frac{\mu_{A}+\mu_{B}}{2}$.

Therefore, our model $\left(E_{1}=E_{2}=0, E_{3} \rightarrow \infty\right)$ is equivalent with a BEG model characterized by a rather unusual set of interaction parameters: $C=0$, a bilinear exchange constant $J=E_{3} / 2 \rightarrow \infty$, and a biquadratic exchange constant $K=-E_{3} / 2 \rightarrow-\infty$ such that $J / K=-1$.

The description in terms of the spin variables $\sigma_{i}$ opens an alternative way of computing the partition function in Eq. (2) by using the standard transfer-matrix method [31]. Although it is possible to set up the transfer matrix using directly the Hamiltonian given in Eq. (26), it turns out to be more convenient to define local fields $\mu\left(\sigma_{i}\right)$ as

$$
\mu\left(\sigma_{i}\right)= \begin{cases}-\mu_{A}, & \text { if } \sigma_{i}=1, \\ +\mu_{B}, & \text { if } \sigma_{i}=-1, \\ 0, & \text { if } \sigma_{i}=0\end{cases}
$$

such that the partition function for the system is written as

$$
Z_{N}^{(B E G)}=\sum_{\left\{\sigma_{i}\right\}} \exp \left[\sum_{i=1}^{N}\left(-\beta J_{i, i+1} \sigma_{i} \sigma_{i+1}-\beta \mu\left(\sigma_{i}\right) \sigma_{i}\right)\right],
$$

or, equivalently, as the trace of the product of transfer matrices

$$
Z_{N}^{(B E G)}=\operatorname{Tr} \prod_{i=1}^{N} V_{i, i+1}
$$

where the transfer matrix $V_{i, i+1}$ is given by

$$
V_{i, i+1}=\exp \left[-\beta J_{i, i+1} \sigma_{i} \sigma_{i+1}-\beta\left(\mu\left(\sigma_{i}\right) \sigma_{i}+\mu\left(\sigma_{i+1}\right) \sigma_{i+1}\right) / 2\right] .
$$


In the thermodynamic limit the expressions for the pressure given by the partition functions in Eqs. (2) and (28) become identical if $E_{1}=E_{2}=0$, and $E_{3} \rightarrow \infty$. For these values of the parameters, the transfer matrix $V_{i, i+1}$ becomes

$$
\mathrm{V}_{0}=\mathrm{V}_{i, i+1}=\left(\begin{array}{ccc}
z_{A} & \sqrt{z_{A}} & 0 \\
\sqrt{z_{A}} & 1 & \sqrt{z_{B}} \\
0 & \sqrt{z_{B}} & z_{B}
\end{array}\right) \text {. }
$$

The eigenvalues $\lambda_{j}=1 / t_{j}$ of $\mathrm{V}_{0}$ are given by $\operatorname{det}\left(V_{0}-\lambda I\right)=0$, which are the inverse of the zeroes of $\mathcal{L}_{2}(t)=0$.

\section{$5 \quad$ Mean density and compressibility}

\subsection{Thermodynamics}

Since the model and the partition sum are symmetric with respect to interchanging $A$ and $B$ particles, it is sufficient to analyze the behavior of only one of the two densities, e.g., $n_{A}$; the corresponding results for $n_{B}$, and similarly for the compressibilities, follow by simply switching the indices $A$ and $B$.

Using Eq. (21), the mean density $n_{A}$ and the compressibility $\varkappa_{A}$ of the phase formed by the adsorbed $A$ particles are given as

$$
n_{A}=\beta z_{A} \frac{\partial P}{\partial z_{A}}, \quad \varkappa_{A}=\frac{z_{A}}{n_{A}^{2}} \frac{\partial n_{A}}{\partial z_{A}} .
$$

The behavior of $n_{A}$ as a function of $z_{A}$ and $z_{B}$, respectively, is shown in Fig. (2) for several values of $z_{B}$ and $z_{A}$, respectively.

As can be seen in Fig. 2, the density $n_{A}$ is very small for $z_{A}<z_{A}^{*}$ (where $\left(z_{A}^{*}, n_{A}^{*}\right.$ ) is the turning point of $n_{A}\left(z_{A}\right)$, occuring for $z_{B}>1$ ), raises sharply to large values for $z_{A}>z_{A}^{*}$ and, independent of the fixed value for $z_{B}$, approaches asymptotically $n_{A}\left(z_{A} \rightarrow \infty ; z_{B}\right) \rightarrow 1$. The functions $z_{A}^{*}\left(z_{B}\right)$ and $n_{A}^{*}\left(z_{B}\right)$ depicted in Fig. 3 show that the value $z_{A}^{*}$ is an almost linear function of $z_{B}$, satisfying $z_{A}^{*}\left(z_{B}\right) \gtrsim z_{B}$, while the density $n_{A}^{*}=n_{A}\left(z_{A}^{*}\left(z_{B}\right), z_{B}\right)$ raises sharply with increasing $z_{B}$ and saturates at the asymptotic value $n_{A}^{*}\left(z_{B} \rightarrow \infty\right) \rightarrow 0.5$. The width $\Delta z_{A}$ of the sharp increase in $n_{A}$ depends weakly on $z_{B}$ and at fixed $z_{B}$ the function $n_{A}\left(z_{A} ; z_{B}\right)$ converges slowly toward the step function $\Theta\left(z_{A}-z_{B}\right)$ with increasing $z_{B}$. In this limit the system exhibits two generic states: an $A$-depleted one $\left(n_{A} \ll 1\right.$, for $\left.z_{A}<z_{A}^{*}\right)$ and an $A$-saturated one $\left(n_{A} \lesssim 1\right.$, for $\left.z_{A}>z_{A}^{*}\right)$, for which the density $n_{A}$ depends only very weakly on $z_{A}$, separated by a narrow transition region centered at $z_{A}=z_{A}^{*}$. Similar conclusions can be drawn regarding the behavior of the mean density $n_{A}$ as a function of $z_{B}$. Comparing 

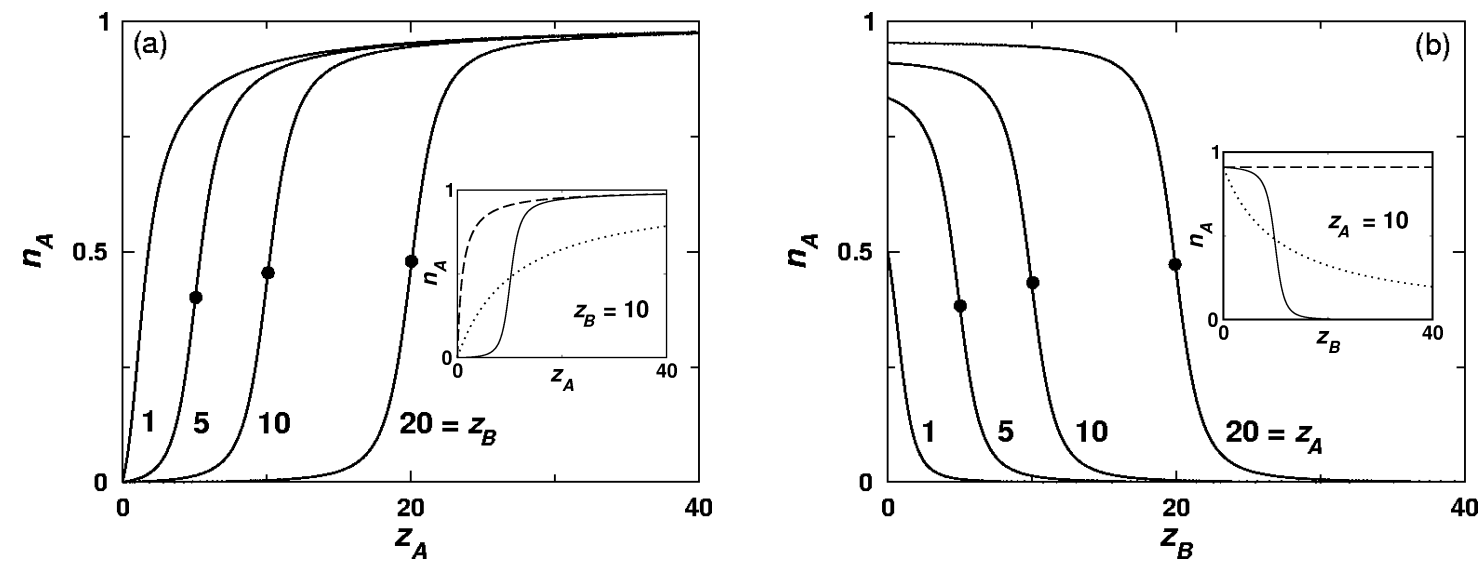

Figure 2: Mean density $n_{A}$ as a function of $z_{A}$ (a) and $z_{B}$ (b), respectively. From left to right, the curves correspond to (a) $z_{B}=1,5,10$, and 20 and to (b) $z_{A}=1,5,10$, and 20, respectively. The dots indicate the turning points $\left(z_{A}^{*}, n_{A}^{*}\right)$ and $\left(z_{B}^{*}, n_{A}^{*}\right)$. For comparison, the insets show results for $z_{B}=10(\mathrm{a})$ and for $z_{A}=10(\mathrm{~b})$, respectively, together with corresponding results for the case of no $A B$ reaction (classical Langmuir, Eq. (5), dashed line) and for the approximation given by Eq. (6) (hard-core exclusion only, dotted line).
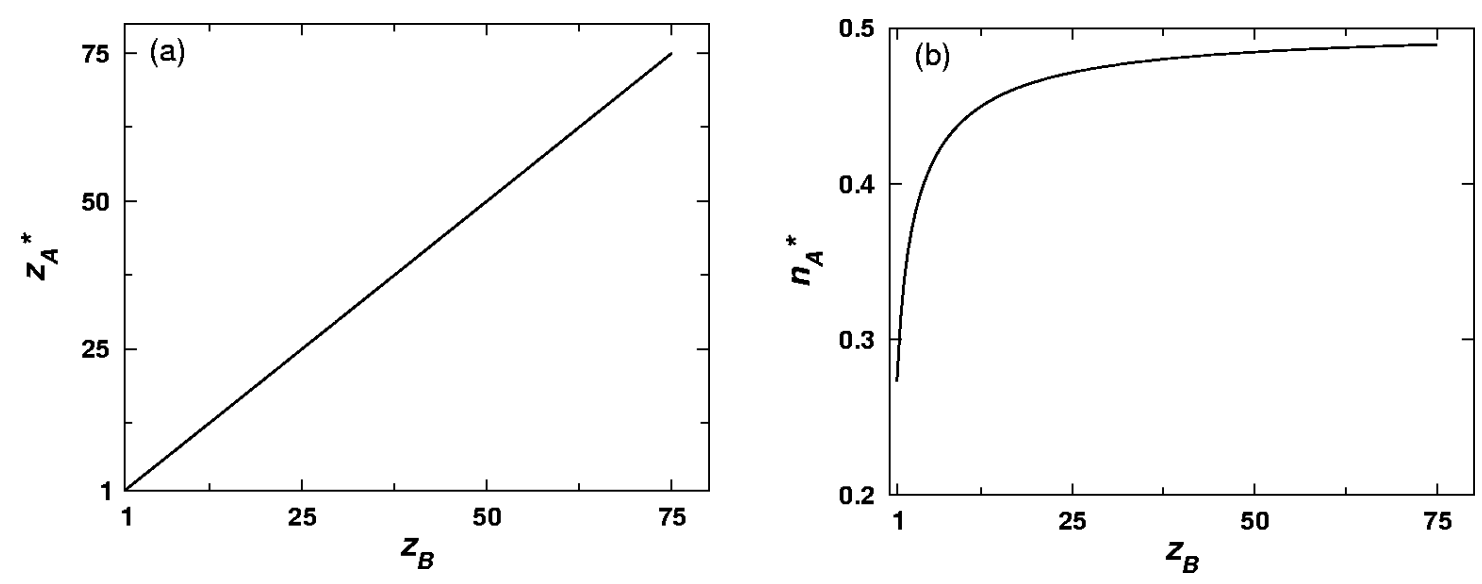

Figure 3: The coordinates $\left(z_{A}^{*}, n_{A}^{*}\right)$ of the turning point of the curves $n_{A}\left(z_{A}\right)$ as functions of $z_{B}$.

these results with the ones corresponding to Langmuir adsorption (Eqs. (5) and (6)) it is evident that in general the reaction $A B$ leads to a qualitatively different behavior of the mean density $n_{A}$ (see the insets in Fig. 2). We note that, as expected, at fixed $z_{A}$ (inset Fig. 2(b)) the behavior for $z_{B} \ll 1$ is well described by the classical Langmuir non-interacting particles model but, rather counter-intuitively, the behavior at large $z_{A}$ and fixed $z_{B}$ (inset Fig 2(a)) is also similar to that of a non-interacting case and not similar to the corresponding case with hard-core interaction. These findings will be addressed in the discussion of the asymptotic 
behavior below.

In Fig. 4 we show the compressibility $\varkappa_{A}$ of the phase formed by the adsorbed $A$ particles as a function of the activities $z_{A}$ and $z_{B}$, respectively, for the same values as in Fig. 2. The
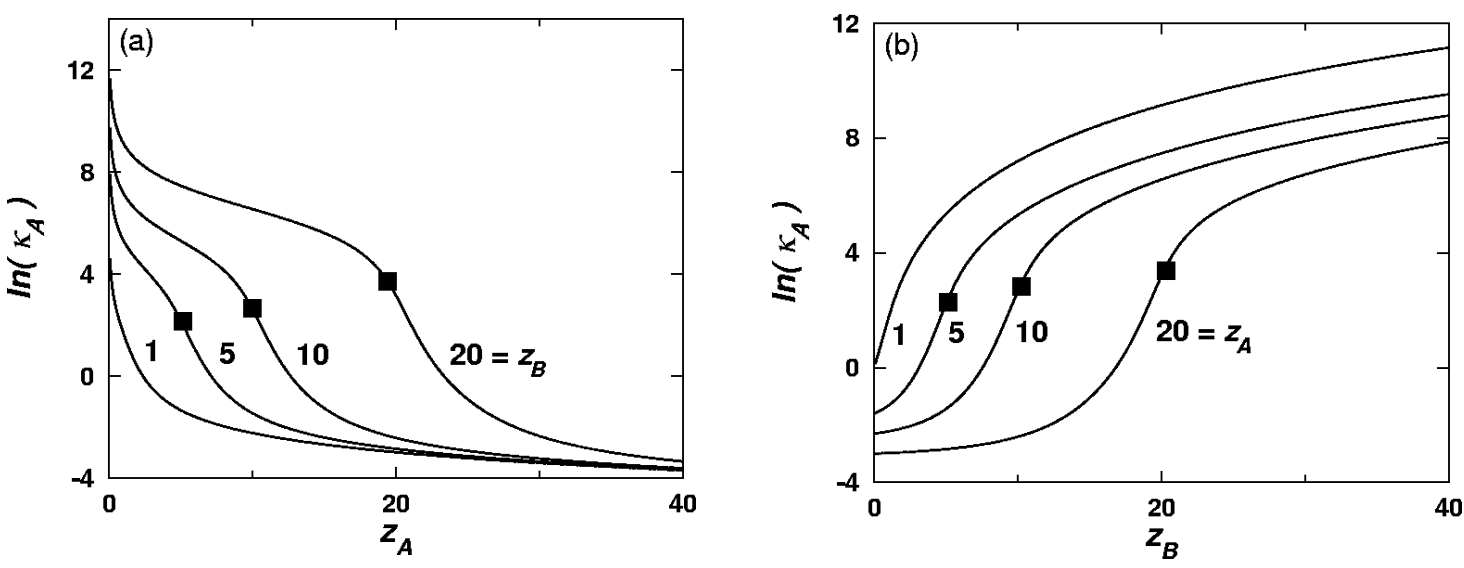

Figure 4: Logarithm of compressibility $\varkappa_{A}$ as a function of $z_{A}$ (a) and $z_{B}$ (b), respectively. From left to right, the curves correspond to (a) $z_{B}=1,5,10$, and 20 and (b) $z_{A}=1,5,10$, and 20, respectively. The squares denote the position of the turning points of $n_{A}\left(z_{A}\right)$ (Fig. 2(a)) and $n_{A}\left(z_{B}\right)$ (Fig. 2(b)), respectively.

behavior of the compressibility is consistent with that of the mean density, i.e., for example Fig. 2(a) shows that the low $A$-density phase at $z_{A}<z_{A}^{*}$ has a large and slowly decreasing compressibility, while the high $A$-density phase at $z_{A}>z_{A}^{*}$ has a very small compressibility.

As indicated by Eqs. (21) and (32), the functions $n_{A}\left(z_{A}, z_{B}\right)$ and $\varkappa_{A}\left(z_{A}, z_{B}\right)$ exhibit complicated dependences on $z_{A}, z_{B}$, and thus an analytical study of the behavior of the system for all the values of the parameters is difficult. However, as we have mentioned, for fixed values of one of the activities, for example $z_{B}$, there are three particular cases as a function of the other one, i.e., $z_{A}$ : $z_{A} \ll z_{A}^{*}, z_{A} \gg z_{A}^{*}$, and $z_{A} \simeq z_{A}^{*}$. Below we derive and discuss the asymptotic behavior in these limits, starting from corresponding expansions of $n_{A}\left(z_{A}, z_{B}\right)$ or, if necessary, of $t_{2}\left(z_{A}, z_{B}\right)$ (Eq. (20)).

\subsection{Asymptotic expansions}

We first consider the case when either one or both activities are small, i.e., the corresponding vapor pressure is low, the temperature is high, or the barrier against desorption is small. In the case when $z_{A} \ll 1$, while $z_{B}$ is fixed, we find from Eqs. (21) and (32):

$$
n_{A}=\frac{1}{\left(1+z_{B}\right)^{3}} z_{A}+\frac{\left(2 z_{B}^{2}+6 z_{B}-1\right)}{\left(1+z_{B}\right)^{6}} z_{A}^{2}+\mathcal{O}\left(z_{A}^{3}\right),
$$


and thus the compressibility is

$$
\varkappa_{A}=\frac{\left(1+z_{B}\right)^{3}}{z_{A}}-\frac{\left(z_{B}^{3}+6 z_{B}^{2}+11 z_{B}+9\right) z_{B}}{\left(1+z_{B}\right)^{3}} z_{A}+\mathcal{O}\left(z_{A}^{2}\right) .
$$

We note that in Eq. (33) the coefficient of the term linear in $z_{A}$ is smaller by a factor $\left(1+z_{B}\right)^{-3}$ than the corresponding one in the case of non-interacting Langmuir adsorption (Eq. (5)) and by a factor $\left(1+z_{B}\right)^{-2}$ compared with hard-core Langmuir adsorption (Eq. (6)). Consequently, the compressibility is increased by similar factors. This reduction of $n_{A}$ is significant for large $z_{B}$ and shows that in the presence of many adsorbed $B$ particles most of the adsorbed $A$ particles do react with $B$ and leave the chain. In the opposite case $z_{B} \ll 1$ the first-order term is the same as for the non-interacting Langmuir adsorption (Eq. (5)) since this limit corresponds to very low densities of both $A$ and $B$ particles and, consequently, very unlikely reaction events.

Second, within the limit $z_{B} \ll 1$, while $z_{A}$ is fixed, we obtain

$$
n_{A}=\frac{z_{A}}{1+z_{A}}-\frac{3 z_{A}}{\left(1+z_{A}\right)^{4}} z_{B}-\frac{\left(4 z_{A}^{2}+13 z_{A}-6\right) z_{A}}{\left(1+z_{A}\right)^{7}} z_{B}^{2}+\mathcal{O}\left(z_{B}^{3}\right)
$$

and

$$
\varkappa_{A}=\frac{1}{z_{A}}+\frac{3\left(3 z_{A}+1\right)}{z_{A}\left(1+z_{A}\right)^{3}} z_{B}+\mathcal{O}\left(z_{B}^{2}\right) .
$$

As expected, the first term in the series for both $n_{A}$ and $\varkappa_{A}$ is the trivial Langmuir adsorption model result (Eq. (5); see also the inset in Fig. 2(b)), because the very low density of adsorbed $B$ particles leads to a very small probability for a reaction $A B$.

Third, for $z_{A}=z_{B}=z \ll 1$ we find

$$
n_{A}=n_{B}=z-4 z^{2}+19 z^{3}+\mathcal{O}\left(z^{4}\right)
$$

and

$$
\varkappa_{A}=\frac{1}{z}+3+3 z-8 z^{2}+\mathcal{O}\left(z^{3}\right)
$$

which shows that at small activities the value of the density $n_{A}$ (or $n_{B}$ ) at the crossover $z_{A}=z_{B}$ increases linearly with the activity.

Next we turn to the case when either one or both of activities are large, which can be realized in systems with low temperature, suppressed desorption, or at high pressures of the corresponding vapor phase. Most of the previous theoretical work has been focused on this limit [23]. Since in this case the analysis is somewhat more complicated compared to the situations with small activities, it is advantageous to start from the asymptotic behavior of $t_{2}$ given in Eq. (20). 
First, we consider the case $z_{A} \gg 1$ and $z_{B}$ fixed. Using the identity

$$
\sin \left(\frac{1}{3} \arcsin \left(\frac{\sqrt{z_{B}}\left(9+2 z_{B}\right)}{2\left(3+z_{B}\right)^{3 / 2}}\right)\right)=\frac{1}{2} \sqrt{\frac{z_{B}}{3+z_{B}}},
$$

one finds for the Laurent series of $t_{2}$

$$
t_{2}=\frac{1}{z_{A}}-\frac{1}{z_{A}^{2}}+\frac{1}{z_{A}^{3}}-\frac{\left(1+z_{B}\right)}{z_{A}^{4}}+\mathcal{O}\left(\frac{1}{z_{A}^{5}}\right) .
$$

This implies for the pressure

$$
\beta P=\ln \left(z_{A}\right)+\frac{1}{z_{A}}-\frac{1}{2 z_{A}^{2}}+\frac{\left(1+3 z_{B}\right)}{3 z_{A}^{3}}+\mathcal{O}\left(\frac{1}{z_{A}^{4}}\right)
$$

and, hence, for the mean density of the $A$ particles

$$
n_{A}=1-\frac{1}{z_{A}}+\frac{1}{z_{A}^{2}}-\frac{\left(1+3 z_{B}\right)}{z_{A}^{3}}+\mathcal{O}\left(\frac{1}{z_{A}^{4}}\right) .
$$

Therefore, in this limit the dependence on $z_{B}$ appears only in the third-order term and thus it is very weak. This explains the confluence of the density curves $n_{A}$ in Fig. 2(a) in the range of large $z_{A}$. Here we also note that regardless of the value of $z_{B}$ (provided that $z_{B} \ll z_{A}$ ) the mean density of the $A$ particles tends to unity, i.e., the one-dimensional chain becomes saturated with $A$ particles. Moreover, the first three terms in the expansion in Eq. (42) coincide with the corresponding terms in the expansion of the mean particle density for large $z_{A}$ in the non-interacting Langmuir adsorption model (Eq. (5)). However, only the first term agrees with the similar expansion of the hard-core Langmuir adsorption (Eq. (6)). This confirms the numerical results shown in the inset in Fig. 2(a) and suggests that the reaction term exactly cancels the contribution of the hard-core interaction up to the third order in $1 / z_{A}$. Intuitively, this can be understood by noting that for $z_{A} \gg z_{B}$ the system tends to a state with a very low density $n_{B}$, and thus the hard-core constraint that a site cannot be occupied simultaneously by an $A$ and a $B$ particle is effectively irrelevant, but the constraint that a site cannot be occupied by two $A$ particles becomes very important. In this limit we obtain for the compressibility

$$
\varkappa_{A}=\frac{1}{z_{A}}+\frac{9 z_{B}}{z_{A}^{3}}+\mathcal{O}\left(\frac{1}{z_{A}^{4}}\right) .
$$

This confirms that in leading order the adsorption is given by the Langmuir-type result for the non-interacting adsorption model with corrections occurring only in third order.

In the opposite limit $z_{B} \gg 1$ and $z_{A}$ fixed, similar calculations lead to

$$
t_{2}=\frac{1}{z_{B}}-\frac{1}{z_{B}^{2}}+\frac{1}{z_{B}^{3}}-\frac{\left(1+z_{A}\right)}{z_{B}^{4}}+\frac{\left(1+4 z_{A}-z_{A}^{2}\right)}{z_{B}^{5}}+\mathcal{O}\left(\frac{1}{z_{B}^{6}}\right),
$$


which implies

$$
n_{A}=\frac{z_{A}}{z_{B}^{3}}-\frac{\left(3-2 z_{A}\right) z_{A}}{z_{B}^{4}}+\frac{\left(6-6 z_{A}+3 z_{A}^{2}\right) z_{A}}{z_{B}^{5}}+\mathcal{O}\left(\frac{1}{z_{B}^{6}}\right),
$$

and, respectively,

$$
\varkappa_{A}=\frac{z_{B}^{3}}{z_{A}}+\frac{3 z_{B}^{2}}{z_{A}}+\mathcal{O}\left(z_{B}\right) .
$$

Thus $n_{A}$ exhibits a very fast power-law decay implying a strong divergence of the compressibility $\varkappa_{A}$ for increasing $z_{B}$.

Lastly, for $z_{A}=z_{B}=z \gg 1$ we find

$$
n_{A}\left(=n_{B}\right)=\frac{1}{2}-\frac{1}{z}+\frac{4}{z^{2}}+\mathcal{O}\left(\frac{1}{z^{3}}\right), \quad \varkappa_{A}=\frac{4}{z}-\frac{16}{z^{2}}+\mathcal{O}\left(\frac{1}{z^{3}}\right) .
$$

\subsection{Monte Carlo simulations}

The result in Eq. (47) tells that in this limit the sites are on average equally occupied by $A$ and $B$ particles. Since these findings give no insight into the actual spatial arrangement, i.e., the correlations, we have performed a simple Monte Carlo simulation. We start with an empty lattice of $N$ sites and update the occupation numbers as follows. At any step, an empty (0) site is picked at random and an $A(+1)$ or a $B(-1)$ particle is deposited with equal probabilities (corresponding to equal activities $z_{A}=z_{B}=z$ ). If any of the two neighboring sites are occupied by a particle of opposite sign, the deposited particle and the neighboring one are removed, i.e., the occupation numbers of the sites are set to 0 . If both of the neighboring sites are occupied by particles from the other species, one of them is randomly selected for desorption. Desorption of individual particles is disregarded since we are considering the limit $z \rightarrow \infty$. Fig. 5 shows typical simulation results for the evolution of the system. The results clearly show the formation of domains consisting of mainly $A$ or $B$ particles such that only a small number, eventually just two in the limit $N \rightarrow \infty$, of large domains emerges. Intuitively, the emergence of two domains is due to the fact the in the limit $z \rightarrow \infty$ the number of empty sites should be minimum since the ratio of the probability of a state with a greater number of empty sites and the probability of the state with minimum number of empty sites goes to zero as $z \rightarrow \infty$. Here we note that for a finite size system increasing the number of updates will eventually lead to only one domain of $A$ or, equally probable, $B$, spanning the whole lattice. This is due to the fact that for $z \rightarrow \infty$ tiny deviations from $z_{A}=z_{B}$, i.e., from exactly equal probabilities of depositing $A$ or $B$, are sufficient to drive the system into one of the $n_{A}=0$ or $n_{A}=1$ states (see also Fig. 2). These results show that in the limit $z_{A}=z_{B} \rightarrow \infty$, the system undergoes, in the thermodynamic limit $N \rightarrow \infty$, segregation in a state in which half of the sites belong to a domain of either $A$ or $B$ particles. Interestingly enough, similar behavior 

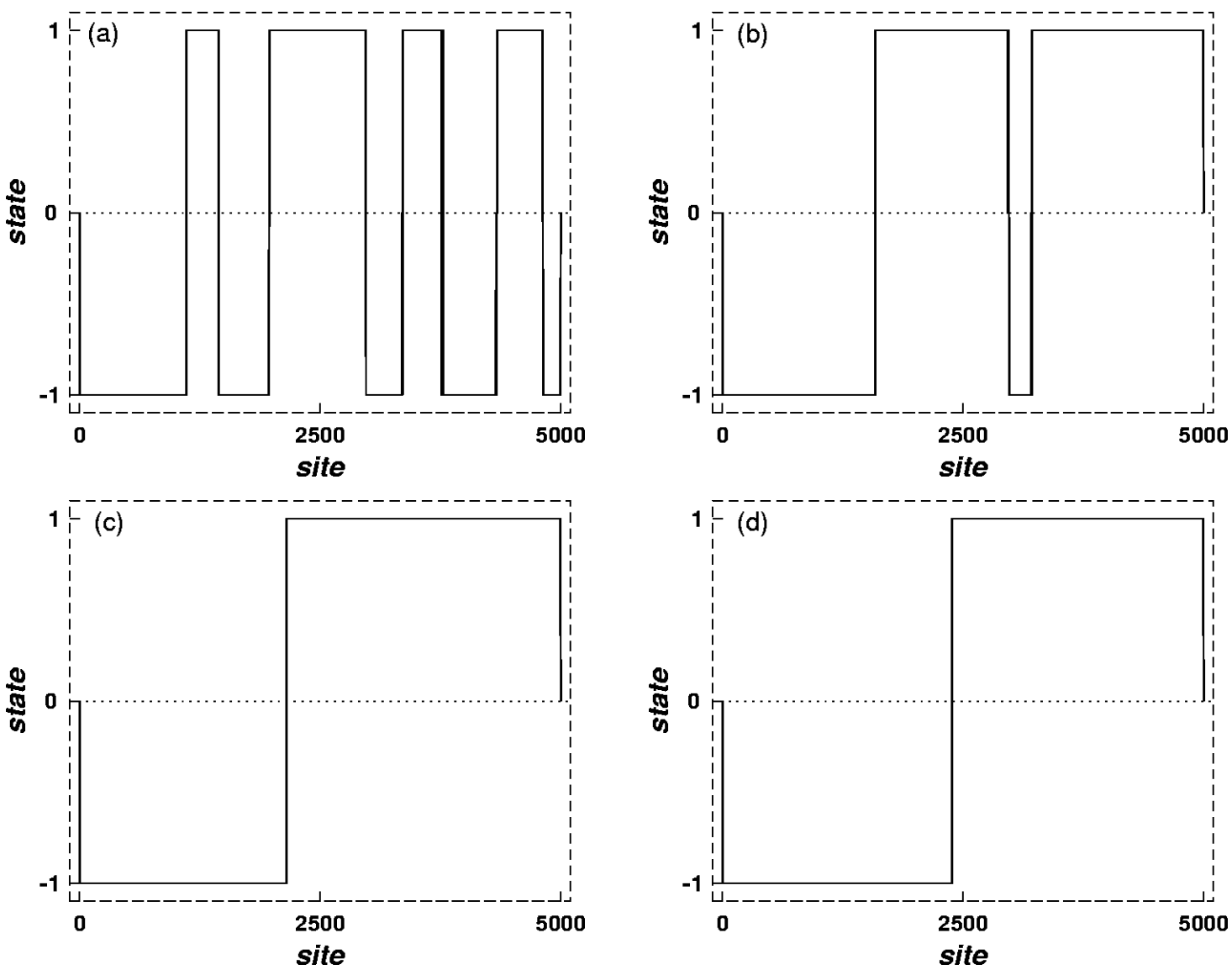

Figure 5: Occupation of a lattice of size $N=5000$ after (a) $2.5 \times 10^{6}$, (b) $5 \times 10^{6}$, (c) $7.5 \times 10^{6}$, and (d) $10^{7}$ updates. Particles of type $A$ and $B$ correspond to +1 and -1, respectively.

has been predicted for the steady-state behavior of diffusion-limited $A+B \rightarrow 0$ reactions in systems with steady injection of the reactive species by external sources with equal intensities $[32,33]$ (for more details see also Ref.[21]).

\section{Conclusions.}

In this paper we have presented a model of monomer-monomer $A+B \rightarrow 0$ catalytic reactions on a one-dimensional chain in contact with a reservoir of $A$ and $B$ particles. The model assumes continuous exchange of $A$ and $B$ species between the chain and the vapor phase acting as a reservoir, and instantaneous reaction and desorption of neighboring $A$ and $B$ particles. We have calculated exactly the partition sum taking into account equilibrium fluctuations. From this we have obtained the pressure of the adsorbed particles, the mean density, and the compressibility. Altough in this one-dimensional model there is no phase- 
transition, the system exhibits a rather non-trivial behavior. In particular, the mean density of the $A$ particles changes rapidly from very small values when the activity $z_{B}$ is larger than the activity $z_{A}$ to a state in which the lattice is occupied predominantly by $A$ particles when $z_{A}$ is larger than $z_{B}$. In the case when the two activities are exactly equal and large the system undergoes segregation such that each of the two species clusters into a large domain and occupies half of sites of the chain.

\section{Acknowledgments}

We thank Prof. P. L. Krapivsky for a critical reading of the manuscript and helpful comments.

\section{References}

[1] R. M. Ziff, E. Gulari, and Y. Barshad, Phys. Rev. Lett. 56, 2553 (1986).

[2] A. Clark, The Theory of Adsorption and Catalysis (Academic, New York, 1970); G.C. Bond, Heterogeneous Catalysis: Principles and Applications (Clarendon, Oxford, 1987); D. Avnir, R. Gutfraind, and D. Farin, in Fractals in Science, edited by A. Bunde and S. Havlin (Springer, Berlin, 1994), vol.2, pp. 229-255.

[3] I. Jensen, H. C. Fogedby, and R. Dickman, Phys. Rev. A 41, 3411 (1990).

[4] R. M. Ziff and K. Fichthorn, Phys. Rev. B 34, 2038 (1986); K. Fichthorn, E. Gulari, and R. M. Ziff, Phys. Rev. Lett. 63, 1527 (1989).

[5] P. Meakin and D. Scalapino, J. Chem. Phys. 87, 731 (1987).

[6] A. Sadiq and K. Yaldram, J. Phys. A 21, L207 (1988).

[7] E. V. Albano, Phys. Rev. Lett. 69, 656 (1992).

[8] J. Zhuo and S. Redner, Phys. Rev. Lett. 70, 2822 (1993).

[9] E. V. Albano, J. Phys. A 25, 2557 (1992); J. Stat. Phys. 69, 643 (1992); H. Zhonghuai, Y. Lingfa, and X. Houwen, Phys. Rev. E 58, 234 (1998).

[10] D. ben-Avraham and J. Köhler, J. Stat. Phys. 65, 839 (1991).

[11] D. Considine, S. Redner, and H. Takayasu, Phys. Rev. Lett. 63, 2857 (1989).

[12] J. Mai, W. von Niessen, and A. Blumen, J. Chem. Phys. 93, 3685 (1990).

[13] E. Clément, P. Leroux-Hugon, and L. M. Sander, Phys. Rev. Lett. 67, 1661 (1991). 
[14] J. W. Evans, Langmuir 7, 2514 (1991); J. W. Evans, J. Chem. Phys. 97, 572 (1992); J. W. Evans and T. R. Ray, Phys. Rev. E 47, 1018 (1993).

[15] P. L. Krapivsky, Phys. Rev. A 45, 1067 (1992); L. Frachebourg, P. L. Krapivsky, and S. Redner, Phys. Rev. Lett. 75, 2891 (1995), P. L. Krapivsky, Phys. Rev. E 52, 3455 (1995).

[16] M. H. Kim and H. Park, Phys. Rev. Lett. 73, 2579 (1994).

[17] R. Dickman, Phys. Rev. A 34, 4246 (1986).

[18] L. Frachebourg and P. L. Krapivsky, Phys. Rev. E 53, R3009 (1996).

[19] D. S. Sholl and R. T. Skodje, Phys. Rev. E 53, 335 (1996).

[20] R. A. Monetti, Phys. Rev. E 58, 144 (1998).

[21] P. Argyrakis, S. F. Burlatsky, E. Clément, and G. Oshanin, Phys. Rev. E 63, 021110 (2001).

[22] F. Vikas, F. Baras, and G. Nicolis, Phys. Rev. E 66, 036133 (2002).

[23] J. Marro and R. Dickman, Nonequilibrium Phase Transitions in Lattice Models (Cambridge University, Cambridge, 1999).

[24] G. Oshanin and S.F. Burlatsky, J. Phys. A 35, L695 (2002); ibid, Phys. Rev. E (in press).

[25] G. Oshanin, O. Benichou, and A. Blumen, submitted to J. Stat. Phys.

[26] G. Oshanin and A. Blumen, J. Chem. Phys. 108, 1140 (1998); S. Toxvaerd, J. Chem. Phys. 109, 8527 (1998).

[27] see, e.g., M. Abramowitz and I. A. Stegun, The Handbook of Mathematical Functions (Dover, New York, 1972).

[28] J. B. Collins, P. A. Rikvold, and E. T. Gawlinski, Phys. Rev. B 38, 6741 (1988).

[29] M. Blume, V. J. Emery, and R. B. Griffiths, Phys. Rev. A 4, 1071 (1971).

[30] D. Furmane, S. Dattagupta, and R. B. Griffiths, Phys. Rev. B 15, 441 (441).

[31] R. J. Baxter, Exactly Solved Models in Statistical Mechanics (Academic, New York, 1982). 
[32] A. A. Ovchinnikov and S. F. Burlatsky, Pis'ma Zh. Eksp. Teor. Fiz. 43, 494 (1986) [JETP Letters 43, 638 (1986)]; S. F. Burlatsky, A. A. Ovchinnikov, and K. A. Pronin, Zh. Eksp. Teor. Fiz. 92, 625 (1987) [Sov. Phys. JETP 65, 353 (1987)].

[33] K. Lindenberg, B. J. West, and R. Kopelman, Phys. Rev. Lett. 60, 1777 (1988); R. Kopelman, Science 241, 1620 (1988). 